\title{
Primary Focal Segmental Glomerulosclerosis, an Important Component of Childhood Nephrotic Syndrome: Therapeutic Options and Genetic Basis
}

\author{
James C.M. Chan ${ }^{*}, 1$ and Karl S. Roth ${ }^{2}$ \\ ${ }^{1}$ University of Vermont College of Medicine, The Barbara Bush Children's Hospital, Maine Medical Center, Portland, \\ Maine, USA \\ ${ }^{2}$ Department of Pediatrics, Creighton University, Omaha, Nebraska, USA
}

\begin{abstract}
Introduction: We aim to review the therapeutic options in ameliorating the progression of focal segmental glomerulosclerosis (FSGS). We shall comment on the long-term prognosis and the clinical implications of candidate genes in familial cases of FSGS.

Materials and Method: Key references from the past concerning FSGS were analyzed, together with a PubMed search of the literature from 1998 to 2008.

Results and Discussion: Treatment of FSGS consists of one or more of the following medications: vitamin E, prednisone, angiotensin-converting enzyme inhibitor, angiotensin receptor blocker; in the severe cases, methylprednisolone, cyclophosphamide, cyclosporine, calcineurin inhibitors, rituximab and mycophenolate. The prognoses with lengths of followup to 14 years were analyzed. The genetics of familial FSGS and the strategy of kidney transplants in such cases need special attention.

Conclusion: We reviewed the available modalities of treatment and the long-term prognosis. The new findings on familial forms of FSGS and the clinical implications are succinctly presented.
\end{abstract}

Keywords: Focal segmental glomerulosclerosis, combined therapy, long-term outcome.

\section{INTRODUCTION}

Glomerular capillary blood is separated from the urinary space by a composite barrier, consisting of the fenestrated endothelium of the vessel itself, the glomerular basement membrane (GBM) and the podocytes, with their foot processes applied to the GBM. Each of the three components is distinctly different from the others, not only in function but in molecular characteristics as well. It is this barrier which permits selective filtration and prevents loss of protein from the several liters of blood flowing through the glomeruli daily. Many pathologic conditions which adversely impact upon this barrier may cause renal disease of variable nature and extent.

The majority of children with nephrotic syndrome respond to steroid therapy and do not suffer long-term consequences of the disease [1]. The underlying renal histologic examination appears normal, which has led to application of the term "minimal change nephrotic syndrome", or MCNS. It is for this reason that it is not standard practice to perform renal biopsy when there is prompt and definitive response to steroid therapy. The minority of patients who fail to respond are likely to suffer from more serious kidney pathology, of which "focal segmental glomerulosclerosis" or "FSGS" is the most likely finding on examination of a kidney biopsy specimen $[1,2]$. Of late, there has been a great deal of

*Address correspondence to this author at the University of Vermont College of Medicine, The Barbara Bush Children's Hospital, Maine Medical Center, 22 Bramhall Street, Portland, Maine 04102-3175, USA; Tel: 207871-2439; Fax: 207-871-6272; E-mail: chanj@suscom-maine.net concern that this minority of patients progressing to FSGS has been growing in proportion to total cases of nephrotic syndrome [3-6]. By contrast, however, in a carefullyconstructed, retrospective study of more than 200 children consecutively diagnosed in a 25 year period and with at least 2 years of follow up, Boyer and colleagues were unable to document any increase in incidence of FSGS in this group of subjects [7]. It may be that FSGS represents a final common pathway of several types of renal disease; in this paper we will focus our attention on the primary forms of FSGS.

\section{MATERIAL AND METHODS}

Key references from the past concerning FSGS, together with information gathered from a PubMed search of the literature from 1998 to 2008, form the basis of this commentary. We have endeavored to summarize the most current and relevant information from the world's literature pertaining to etiologies, treatment modalities and prognosis of this major cause of morbidity.

\section{RESULTS AND DISCUSSION}

Histology: In FSGS, segments of the glomerulus are sclerotic, giving rise to the descriptor: segmental glomerulosclerosis [8]. In view of the fact that focal areas of the kidney's cortex, mostly the cortico-medullalry junction, show evidence of these damaged glomeruli whereas other areas show normal appearing glomeruli, the descriptor focal is used to come up with the full name focal segmental glomerulosclerosis or FSGS. However, a working pathological classification [9] for FSGS was not available until 2004. If this pathological classification for FSGS becomes universally 
accepted, the progression to end-stage kidney disease (ESKD) may be shown to vary dependent on the histological classification of collapsing, tip, cellular, perihilar and "not otherwise specified" or NOS variant of FSGS [9]. The collapsing lesions are more common in black children, in whom the incidence of FSGS is acknowledged to be substantially increased. The tip lesions are more common in Caucasian children and are associated with less tubulointerstitial injury and better glomerular filtration rates. It should be kept in mind, however, that the biopsied patients are a selected population whose pre-biopsy histological features from the inception of their disease remain unknown.

Racial and Gender Disposition: FSGS is the underlying pathology in up to $10 \%$ of children with the nephrotic syndrome $[1,2,8]$. The clinical impression that the incidence is probably higher in African-American and Asian children is bolstered by the recent findings that in South Africa, FSGS is much higher in African and in Asians, at incidences of $27 \%$ and $20 \%$ respectively for black and Asian children [10]. It should be noted that the Asian children studied in this report are South Asian, primarily from the Indian subcontinents. A comparison with Asian children of Chinese, Korean or Japanese heritages has not been published, but there is no indication that this higher incidence would pertain to these children, as well. Gender distribution in FSGS is 2:1 male to female [1, 11]. However, in African-American children with FSGS, there is an equal gender distribution [12].

Clinical Features: The edema of a nephrotic child often presents insidiously, from puffiness of the eyelids to pitting edema of the lower extremities recurring over several weeks until persistent generalized edema brings the child to medical attention $[1,11]$. In minimal change nephrotic syndrome, the child usually presents between 3 to 6 years of age, whereas in FSGS the age of presentation is usually older [2, 8]. Most notably, in African and Asian children, the age of presentation is frequently later than in white children, often after the age of 10 years [12]. It is also not unusual for 30 to $40 \%$ of the children with FSGS to present with hypertension, with diastolic blood pressure exceeding $80 \mathrm{~mm} \mathrm{Hg}[2,12]$. Due to the hypovolemia attendant on massive proteinuria, children would be expected to be either normo- or hypotensive; consequently, hypertension is an ominous sign in a patient who is not already on large doses of steroids.

Laboratory Features: Nephrotic syndrome is clinically characterized by heavy proteinuria of over $40 \mathrm{mg}$ per meter ${ }^{2}$ body surface area per hour in timed urine collection (or urinary albumin to urinary creatinine ratio in excess of 4 $\mathrm{mg} / \mathrm{mg}$ ) $[1,11]$. This heavy protein loss eventually causes a decrease in the serum albumin concentration to less than 2.5 $\mathrm{gm} / \mathrm{dl}$ from the usual normal values of $4 \mathrm{gm} / \mathrm{dl}$. It is this loss of serum oncotic pressure which leads eventually to the generalized edema which characterizes the clinical picture. Frequently, there is a total hypocalcemia present, which is most often a consequence of the hypoalbuminemia. The serum cholesterol in MCNS is usually markedly elevated above the normal values; in contrast FSGS patients usually have only mildly elevated serum cholesterol $[1,2,8]$. The mechanism(s) of the hypercholesterolemia so characteristic of MCNS are incompletely understood; however, they include decreased hepatic VLDL-cholesterol catabolism and increased LDL-cholesterol synthesis. Those cause underlying the same laboratory finding in FSGS would seem to be either different in nature or degree. Similar to MCNS, patients with FSGS also have normal serum complement concentrations. This feature distinguishes FSGS from conditions which are hypocomplementemic such as idiopathic membranoproliferative glomerulonephropathy or nephrotic syndrome secondary to systemic diseases such as lupus erythematosus.

Kidney Biopsy: If a child presenting with nephrotic syndrome for the first time is at or above 10 years of age, a kidney biopsy is recommended to rule out FSGS [12]. A child younger than 10 years of age at the time of presentation should not be considered a candidate for a kidney biopsy, because the likelihood of MCNS is high in this age group. However, if there is no response to a course of steroid therapy or if the child has more than 2 relapses in any 6 month period, a kidney biopsy is indicated to rule out FSGS [1, 12]. If nephrotic syndrome presents in the first 12 months of life, a kidney biopsy is indicated, to confirm the diagnosis of congenital nephrotic syndrome [1]. Our understanding of the genetics this so-called "congenital" entity has grown substantially in recent years, and is discussed below. Suffice it to say at this juncture that consideration should be given in all such cases to genotyping the biopsied tissue.

Treatment: If the serum albumin is less than $2 \mathrm{gm} / \mathrm{dl}$, symptoms of hypovolemia (hypotensive abdominal pain, tachycardia, poor capillary refilling, cold extremities) are detected or the edema is so excessive as to increase the risk of skin rupture, the nephrologist or intensivist may choose to use intravenous albumin. In such cases, the usual procedure is to administer a $20 \%$ salt- poor albumin solution at a dose of $1 \mathrm{gm} / \mathrm{kg}$ over a $4-6$ hour interval followed by intravenous furosemide $(1-3 \mathrm{mg} / \mathrm{kg} / \mathrm{dose})$ [1, 11]. If the serum albumin is better than $2 \mathrm{gm} / \mathrm{dl}$ and clinical symptoms of hypovolemia are not present, furosemide alone or with a potassium sparing diuretic spironolactone $(2-4 \mathrm{mg} / \mathrm{kg} /$ day $)$ may be used to promote diuresis and reduce edema. It should be noted that, as renal perfusion improves from intravascular volume expansion, there is mobilization of accumulated extravascular fluid and electrolytes; any presenting hyponatremia may be dilutional in nature, so electrolyte therapy should take this into account. Moreover, frequent weights are essential to monitoring fluid loss, together with twenty-four hour urine volumes, which tend to be inaccurate in young children.

After tuberculosis is ruled out by skin testing, prednisone at $2 \mathrm{mg}$ per $\mathrm{kg}$ body weight per day $\left(60 \mathrm{mg} / \mathrm{meter}^{2}\right.$ body surface areas per day), up to a maximal dose of $80 \mathrm{mg}$ per day, is initiated for 6 weeks, switching to alternative day dosage $\left(1.5 \mathrm{mg}\right.$ per kg every other day or $40 \mathrm{mg} / \mathrm{meter}^{2}$ every other day) for an additional 6 weeks $[1,13]$. The definition of what comprises a complete remission remains problematic; it is generally accepted that, if the child has not completely cleared the urine of protein for 3-5 consecutive days after four weeks of prednisone, remission is incomplete. Some authors consider that a patient with two or more relapses during steroid tapering or within a month thereafter constitutes steroid-dependency [14]. These patients are classified as frequent relapsing/steroid-dependent. Patients with proteinuria persisting beyond three months are considered steroid resistant. If medically stable, these children should be considered candidates for diagnostic renal biopsy. 
If there is no clearing of the nephrotic proteinuria and edema and the kidney biopsy confirms the diagnosis of FSGS, most nephrologists will continue on the alternative dose of prednisone and add an angiotensin converting enzyme inhibitor (lisinopril $0.07 \mathrm{mg} / \mathrm{kg} /$ day, maximal 5 $\mathrm{mg}$ /day) [8,11]. Alternatively, an angiotensin receptor blocker (losartan $0.7 \mathrm{mg} / \mathrm{kg} /$ day, maximal dose $50 \mathrm{mg} / \mathrm{day}$ ) is used in place of the angiotensin converting enzyme inhibitor. Clinical studies in adult patients [15] showed that the combined treatment with both angiotensin converting enzyme inhibitor and receptor blocker resulted in significantly better prognosis. Side effects were minimal and did not exceed those of either drug alone. Currently, however, no data are available in children; perhaps the risk of hypotensive side effects with such combined therapy is a cause of the hesitancy to use this combined drug approach to therapy.

Addition of an antioxidant, specifically Vitamin E (400 IU/day) [16] has also been recommended as an adjunct therapy, because experimental data $[17,18]$ and preliminary clinical studies [19] indicated efficacy in reducing proteinuria and improved prognosis. The risk/benefit ratio of this treatment modality is so low that, while its efficacy remains unproven there is little reason not to add it to the therapeutic regimen. However, this conservative approach to treatment in a great many patients with FSGS usually does little to cause abatement of the proteinuria or disease progression.

In the FSGS cases which are poorly- or unresponsive to the outlined combined treatment strategy, the use of cytotoxics and other immunosuppressive therapies needs to be considered. The decision to move forward with this modality of treatment, however, should be made carefully, because of the significant side effects of such medications. Most pediatric nephrologists will continue the low dose, alternative day prednisone $(1.5 \mathrm{mg} / \mathrm{kg}$ every other day or $40 \mathrm{mg} / \mathrm{square}$ meter every other day) $[1,11]$ but may add cyclophosphamide $(2 \mathrm{mg} / \mathrm{kg} /$ day) [21, 22]; the rationale for this is the addition of the immunosuppressant effects of the latter. Although experience with cyclosporin A (5 mg/kg/day) [22] or levamisole $(2.5 \mathrm{mg} / \mathrm{kg}$ every other day) [23] is still relatively limited and anecdotal, their use is based on experimental nephrotic animal models, in which it is thought that basement membrane damage is mediated by lymphokines. Cyclosporin A is an immunomodulator which acts on T-helper cells, causing decreased interleukin-2 production. Levamisole is no longer available in the United States. However, that lymphokine mediation is the basic mechanism for either the membrane damage or its metamorphosis into FSGS remains inconclusive. The use of intravenous methylprednisolone at varying doses has also been advocated as an alternative to oral steroids, chiefly due to the relatively poor response to oral dosing $[24,25]$. The results are pending on several clinical trials using mycophenolate mofetil (MMF) [26, 27], sirolimus (Rapamycin) [28], tacrolimus (Prograf) [29] and rituximab [30]. The first three drugs are immunosuppressant agents acting on T-cells and impairing lymphokine response, whereas rituximab acts on B-cells to achieve a similar effect. Rituximab is an anti-CD20 monoclonal antibody which is believed to inhibit B-lymphocyte activation, and is currently approved for use in B-cell lymphoma and rheumatoid arthritis. Following a number of sporadic case reports indicating successful use of rituximab in treatment of steroid-dependent nephrotic syndrome, Guigonis and colleagues [31] completed a multicenter study in which they documented the efficacy of the drug as a treatment modality. The success of rituximab treatment in this context has now begun to be extrapolated to treatment of FSGS, although to date only scattered case reports currently exist in the literature [32,33]. A major deficiency of each therapeutic modality is the implicit assumption of an inflammatory mechanism as a primary etiology for FSGS, an assumption without solid evidence other than the rapid response of the vast majority of MCD to large steroid doses [30]. However, we lack among other things, the absolute knowledge that MCD and FSGS are or are not related; hence, to extend a therapeutic philosophy from success with one entity to treatment of another, perhaps unrelated one could be at the basis for the poor overall therapeutic response of FSGS.

The side effects of prednisone and methylprednisolone are euphoria, mood swings, headaches, acne, hirsutism and cushingnoid facial appearance, hypertension, arrhythmia, and increased risk of infections $[1,8]$. Long term use of steroids give rise to cataracts, osteoporosis, short stature $[1,11]$. The side effects of cyclophosphamide are leucopenia from bone marrow suppression, hemorrhagic cystitis, and sterility $[1,11]$. The potentially increased risk of malignancy is unresolved. Cyclosporine $\boldsymbol{A}$ is associated with pruritis, gum hyperplasia, hypertrichosis, neurological complications from confusion to seizures, gastrointestinal complications of nausea, vomiting, diarrhea, pancreatitis, peptic ulcers; hypertension, nephrotoxicity, hyperkalemia and other drug toxicities and interactions. Levamisole causes allergic reactions, abdominal pain, nausea, and vomiting, neurologic complications from confusions to memory loss and seizures, bone marrow suppression and bleeding disorders [22]. The serious side effects of mycophenolate mofetil [11] include headaches, coughing, fatigue, thrombophlebitis, cytomegalovirus and opportunistic infections, anemia, leucopenia from bone marrow suppression and gastrointestinal hemorrhage and perforation. Pregnant women should not take this medication because of fetal risks. Sirolimus side effects $[1,11,27]$ include allergic dermatitis, acne, peripheral edema, hypertension, arthralgia, gastrointestinal upsets, elevated serum triglycerides, cholesterol and creatinine, reduced glomerular filtration rate and proteinuria, and susceptibility to infections. When jointly used with cyclosporine, there is an increased risk of hemolytic uremia syndrome, thrombocytopenic purpura and microangiopathy. The side effects of tacrolimus $[1,11,28]$ include increased risk of hepatotoxicity and nephrotoxicity, hyperglycemia, diabetes mellitus, hypertension, neuropathy, blurred vision, tremors, seizures, confusion, insomnia and herpes zoster infections. The question of cancer risks is not settled. Clearly, in using these compounds for treatment of FSGS, it is essential to give very serious consideration to the risk/benefit ratio and to how their use could adversely impact the quality of life for a patient in whom this quality may already be significantly compromised.

Prognosis in FSGS: When FSGS was first recognized as a distinct kidney disease, steroid therapy was perceived as ineffective and not recommended [34, 35] because the side effects were considered to outweigh the benefits. However, low dose alternate day steroid therapy was later found to improve the prognosis in childhood FSGS, especially when combined with a cytotoxic medication such as cyclophos- 
phamide [20]. Data on long term prognosis of prednisone plus cyclophosphamide showed kidney survival of $60 \%$ at 15 years of follow up in the Toronto study [20]. In the 1980's, intravenous, pulse methylprednisolone [24] over long periods was recommended on the basis of significantly improved kidney survival. However, wide acceptance of this form of therapy did not materialize as additional studies did not support the anticipated improvement reported in the initial study [24]. Low dose prednisone plus angiotensin converting enzyme inhibitor with and without cytotoxic medications combined with vitamin $\mathrm{E}$ were used with the long term prognosis remaining at $50 \%$ kidney survival at 12 years of follow-up in a Virginia study [12]. However, in new clinical studies [36] patients who responded initially to the combined therapy especially with cyclosporine, the prognosis at 4 years was $90 \%$ kidney survival. By contrast, in those who did not respond initially, the prognosis was poor with kidney survival of $40 \%$ at 4 years of follow up [36, 37]

The matter of renal transplantation deserves mention in the treatment of the renal failure induced by FSGS. The North American Cooperative Study documented a 30-40\% incidence of recurrence of FSGS in the graft kidney, with a recurrence rate of about $80 \%$ in a subsequent graft [38]. A more recent report of 10 patients receiving up to four sequential transplants showed a recurrence rate of 100\% [39]. Consequently, efforts are directed at preservation of the initial graft and subsequent renal transplantation is discouraged. These efforts have included a variety of immunosuppressive agents, most already mentioned in our discussion of treatment, but the only combination which seems to be effective in delaying (but not preventing) eventual recurrent FSGS is plasmapheresis plus high-dose cyclosporine, mycophenolate or cyclophosphamide [40]. Controversy in the reported literature over the effectiveness of rituximab in this clinical situation remains to be resolved [41-43]. In general, the differences discussed above in prognosis related to therapeutic modality reflects again the vast ignorance from which we suffer regarding etiology. It is possible, for instance, that the cyclosporine-responders reported to show extended kidney survival suffer from FSGS of a different etiology from those who do not respond. Until we are able to uncover the causal basis for FSGS, prognostication will remain a matter of phenomenology.

Genetics in FSGS: As in a multitude of other entities in medicine, it has become clear that FSGS, initially described histopathologically as a single entity, now comprises a number of distinct disorders, many known to be of clear genetic etiology. Such etiologic diversity is entirely consistent with the differing responses to therapy from patient to patient. It is also predictable, given the diverse molecular composition of the filtration barrier, as pointed out in the Introduction (supra vide). Advances in the past decade on genetic markers in familial FSGS have uncovered several mutations which result in the clinical picture.

Inasmuch as the clinical definition of FSGS includes steroid-unresponsiveness of the proteinuria in a nephrotic patient, congenital or primary nephritic syndrome exemplifies a genetic abnormality resulting in unremitting and massive proteinuria. The underlying cause is a mutation of the nephrin gene (NPHS1); nephrin is a protein localized to the slit between foot processes and forms a network which lines the pores which, are in turn about equal in size to albumin molecules [44]. Thus, it is not difficult to understand why a genetic mutation inducing abnormality in this complex structure would create steroid-resistance. The genetic abnormality is inherited as an autosomal recessive trait and can be definitively treated only by renal transplant.

Frishberg and colleagues [45] studied a kindred in which there was familial incidence of FSGS. These investigations, utilizing positional cloning technique showed mutation in the NPHS2 gene which encodes the podocin protein. Podocin is an integral membrane protein which binds to cholesterol in the podocyte membrane; it is necessary for interaction with nephrin and facilitates podocytic regulation of the slit diaphragm [46]. Mutation of the NHPS2 gene is linked to autosomal recessive steroid resistant FSGS [45]. With increasing interest and experience in genotyping FSGS patients, it has become clear that there are several NPHS2 mutations at different loci and that collectively these accounts for a significant percentage of both sporadic and familial disease [47, 48].

Alpha-actinin-4(ACTN4) is a protein which binds actin into bundles and is key to cytoskeletal integrity. Mutation of the ACTN4 gene in autosomal dominant FSGS is associated with presentation of renal disease in adolescence and a high risk of progression to end-stage kidney diseases [49]. However, penetrance is variable, so that more mildly-affected individuals can be seen, as well. Another genetic cause of adolescent presentation of FSGS is mutation in the transreceptor potential cation channel (TRPC6) gene. The TRCP6 protein is involved in regulation of calcium entry into the cell and interacts at the slit diaphragm complex with nephrin and podocin. The gene mutation is linked to an autosomal dominant form of FSGS [50]. At present, it is unclear how such gene mutations relate to responses to therapy.

In view of the familial forms of FSGS and the risks of recurrence of FSGS in the transplanted allografts, liverelated donors need to be carefully worked up, including at least one year of investigation on the prospective related donors to ensure that there is no evidence of proteinuria and/or hematuria which may resolve and recur - hence, the recommendation to repeated check urinalysis over a 12 month period as a work up to transplant donation. There are also nephrologists advocating to halt using live-related donors entirely in FSGS, chiefly because of the potential for genetic mutation in the donated kidney.

\section{CONCLUSIONS}

FSGS is an important component of MCNS in childhood. New data demonstrate a higher predisposition in African and Asian children [10] for development of FSGS and an average age of presentation older than 10 years. There is equal gender distribution in the African-American child [12], in contrast to the previous data of male predominance in the Caucasian child with FSGS $[1,2]$. The current treatment of choice is to slow the rate of progression [51], by a combination of vitamin E [18], prednisone [1], angiotensin converting enzyme inhibitor or angiotensin receptor blocker [14]. In the non-responsive cases, the addition of cyclosporine is supported by results of controlled clinical trials [22]. Newer immunosuppressives are undergoing controlled clinical trials. 
The clinical implications of genetic markers are under intense investigation.

\section{ACKNOWLEDGEMENT}

Supported by National Institutes of Health Grants: DK50419, DK07761.

\section{REFERENCES}

[1] Roth KS, Amaker BH, Chan JCM. Nephrotic syndrome. Pediatr Rev 2002; 23: 237-48.

[2] A report of the International Study of Kidney Disease in Children. The primary nephrotic syndrome in children. Identification of patients with minimal change nephrotic syndrome from initial response to prednisone. J Pediatr 1981; 98: 561-4.

[3] Eddy AA, Symons JM. Nephrotic syndrome in childhood. Lancet 2003; 362: 629-39.

[4] Kitiyakara C, Kopp JB, Eggers P. Trends in the epidemiology of focal segmental glomerulosclerosis. Semin Nephrol 2003; 23: 17282.

[5] Srivastava T, Simon AD, Alon US. High incidence of focal segmental glomerulosclerosis in nephritic syndrome of childhood. Pediatr Nephrol 1999; 13: 13-18.

[6] Haas M, Meehan SM, Karrison TG, et al. Changing etiologies of unexplained adult nephritic syndrome: a comparison of renal biopsy findings from 1976-1979 and 1995-1997. Am J Kidney Dis 1999; 30: 621-31

[7] Boyer O, Moulder JK, Somers MJG. Focal and segmental glomerulosclerosis in children: a longitudinal assessment. Pediatr Nephrol 2007; 22: 1159-66.

[8] Niaudet P. Steroid resistant idiopathic nephrotic syndrome in children. In: Avner ED, Harmon WE, Niaudet P, Eds. Pediatric Nephrology. Philadelphia: Lippincott Williams and Wilkins 2004; pp. 557-73.

[9] D'Agati VD, Fogo AB, Bruijn JA, et al. Pathologic classification of focal segmental glomerulosclerosis: a working proposal. Am J Kidney Dis 2004; 43: 368-82.

[10] Adhikari M, Bhimma R, Coovadia HM. Focal segmental glomerulosclerosis in children from KwaZulu/Natal, South Africa. Clin Nephrol 2001; 55: 16-24.

[11] Lee EKW, Chan WKY, Lai WM, et al. Nephrotic syndrome: long term management. In: Chiu MC, Yap HK, Eds. Practical Paediatric Nephrology. An update of current practices. Hong Kong: Medcom Ltd. 2005; pp. 116-29.

[12] Chan JCM. Focal segmental glomerulosclerosis: a single center study over two decades. World J Pediatr 2007; 3: 260-4.

[13] Latta K, von Schnakenburg C, Ehrich JH. A meta-analysis of cytotoxic treatment for frequently relapsing nephrotic syndrome in children. Pediatr Nephrol 2001; 16: 271-82.

[14] Letavernier B, Letavernier E, Leroy S, et al. Prediction of highdegree steroid dependency in pediatric idiopathic nephrotic syndrome. Pediatr Nephrol 2008; 23: 2221-26.

[15] Linas SL. Are two better than one? Angiotensin-converting enzyme inhibitors plus angiotensin receptor blockers from reducing blood pressure and proteinuria in kidney disease. Clin J Am Soc Nephrol 2008; 3: S17-23.

[16] Thabet MA, Chan JCM. Vitamin E in renal treatment regimens. Pediatr Nephrol 2006; 21: 1790-1801.

[17] Hahn S, Kuemmerle NB, Chan W, et al. Glomerulosclerosis in the remnant kidney rat is modulated by dietary $\alpha$-tocopherol. J Am Soc Nephrol 1998; 9: 2089-95.

[18] Lee HS, Jeong JY, Kim BC, et al. Dietary antioxidant inhibits lipoprotein oxidation and renal injury in experimental focal segmental glomerulosclerosis. Kidney Int 1997; 51: 1151-9.

[19] Tahzib M, Frank R, Gauthier B, et al. Vitamin E treatment of focal segmental glomerulosclerosis: results of an open-label study. Pediatr Nephrol 1999; 13: 649-52.

[20] Arbus GS, Poucell S, Bacheyle GS, et al. Focal segmental glomerulosclerosis with idiopathic nephrotic syndrome: three types of clinical response. J Pediatr 1982; 101: 40-5.

[21] Report of Arbeitsgemeinschft fur Padiatrische Nephrologie. Cyclophosphamide treatment of steroid dependent nephrotic syndrome: comparison of eight week with 12 week course. Arch Dis Child 1987; 62: 1102-6.
[22] Lieberman EV, Tejani A. A randomized double-blind placebocontrolled trial of cyclosporine in steroid-resistant idiopathic focal segmental glomerulosclerosis in children. J Am Soc Nephrol 1996; 7: 56-63.

[23] Bagga A, Sharma A, Scrivastava RN. Levamisole therapy in corticosteroid-dependent nephrotic syndrome. Pediatr Nephrol 1997; 11: 415-7

[24] Tune BM, Kirpekar R, Sibley RK, et al. Intravenous methylprednisolone and oral alkylating agent therapy of prednisone-resistant pediatric focal segmental glomerulosclerosis: a long-term follow-up. Clin Nephrol 1995; 43: 84-8.

[25] Waldo FB, Benfield MR, Kohaut EC. Therapy of focal and segmental glomerulosclerosis with methyl prednisolone, cyclosporine A, and prednisone. Pediatr Nephrol 1998; 12:397-400.

[26] Barletta GM, Smoyer WE, Bunchman TE, et al. Use of mycophenolate mofetil in steroid-dependent and -resistant nephrotic syndrome. Pediatr Nephrol 2003; 18: 833-7.

[27] Montane B, Abitbol C, Chandar J, et al. Novel therapy of focal glomerulosclerosis with mycophenolate and angiotensin blockade. Pediatr Nephrol 2003; 18: 772-81.

[28] Tumulin JA, Miller D, Near M, et al. A prospective, open-label trial of sirolimus in the treatment of focal segmental glomerulosclerosis. Clin J Am Soc Nephrol 2006; 1: 109-17.

[29] Segarra A, Vila J, Pou L, et al. Combined therapy of tacrolimus and corticosteroids in cyclosporin-resistant or -dependent idiopathic focal glomerulosclerosis: a preliminary uncontrolled study with prospective follow-up. Nephrol Dial Transplant 2002; 17: 655-62.

[30] Dotsch J, Muller-Wiefel D, Kemper MJ. Rituximab: Is replacement of cyclophosphamide and calcineurin inhibitors in steroiddependent nephrotic syndrome possible? Pediatr Nephrol 2008; 23 3-7.

[31] Guigonis V, Dallocchio A, Baudouin V, et al. Rituximab treatment for severe steroid- or cyclosporine-dependent nephrotic syndrome: a multicentric series of 22 cases. Pediatr Nephrol 2008; 23: 126979.

[32] Nakayama M, Kamei K, Nozu K, et al. Rituximab for refractory focal segmental glomerulosclerosis. Pediatr Nephrol 2008; 23: 48185 .

[33] Suri M, Tran K, Sharma A, et al. Remission of steroid-resistant nephrotic syndrome due to focal and segmental glomerulosclerosis using rituximab. Int Urol Nephrol 2008; 40: 807-10.

[34] Mathieson PW. Minimal change nephropathy and focal segmental glomerulosclerosis. Semin Immunopathol 2007; 29: 415-26.

[35] Habib R. The major syndromes. In: Royer P, Habib R, Mathieu H, Broyer M, Eds. Pediatric Nephrology. Philadelphia: WB Saunders Co. 1974; pp. 246-90.

[36] Gipson DS, Gibson K, Gipson PE, Watkins S, Moxey-Mims M. Therapeutic approach to FSGS in children. Pediatr Nephrol 2007; 22: $28-36$.

[37] Chan JCM. Oxidative injury in focal segmental glomerulosclerosis Asian Biomed 2008; 2(1): 19-26.

[38] Tejani A, Stablein DH. Recurrence of focal segmental flomerulosclerosis posttransplantation: a special report of the North American Pediatric Renal Transplant Cooperative Study. J Am Soc Nephrol 1992; 2(12 Suppl): S258-63.

[39] Jungraithmayr TC, Bulla M, Dippell J, et al. Primary focal segmental glomerulosclerosis - Long-term outcome after pediatric renal transplantation. Pediatr Transplant 2005; 9: 226-31.

[40] Crosson JT. Focal segmental glomerulosclerosis and renal transplantation. Transplant Proc 2007; 39: 737-43.

[41] Gossman J, Scheuermann E-H, Porubsky S, et al. Abrogation of nephrotic proteinuria by rituximab treatment in a renal transplant patient with relapsed focal segmental glomerulosclerosis. Transplant Int 2007; 20: 558-52.

[42] Hristea D, Hadaya K, Marangon N, et al. Successful treatment of recurrent focal segmental glomerulosclerosis after kidney transplantation by plasmapheresis and rituximab. Transplant Int 2007; 20: $102-5$

[43] Yabu JM, Ho B, Scandling JD, et al. Rituxima failed to improve nephrotic syndrome in renal transplant patients with recurrent focal segmental glomerulosclerosis. Am J Transplant 2008; 8: 222-27.

[44] Wartiovaara J, Ofverstedt LG, Khoshnoodi J, et al. Nephrin strands contribute to a porous slit diaphragm scaffold as revealed by electron tomography. J Clin Invest 2004; 114: 1475-83. 
[45] Frishberg Y, Rinat C, Megged O, et al. Mutations in NPHS2 encoding podocin are prevalent cause of steroid-resistant nephrotic syndrome among Israel-Arab children. J Am Soc Nephrol 2002; 13: 400-05.

[46] Huber TB, Schermer B, Muller RU, et al. Podocin and mec-2 bind cholesterol to regulate the activity of associated ion channels. Proc Natl Acad Sci USA 2006; 103: 17079-86.

[47] Berdeli A, Mir S, Yavascan O, et al. NPHS2 (podocin) mutations in Turkish children with idiopathic nephritic syndrome. Pediatr Nephrol 2007; 22: 2031-40.

[48] Hinkes BG, Mucha B, Vlangos CN, et al. Nephrotic syndrome in the first year of life: Two thirds of cases are caused by mutations in
4 genes (NPS1, NPHS2, WT1, and LAMB2). Pediatrics 2007; 119: e907-e19.

[49] Kaplan JM, Kim SH, North KN, et al. Mutation in ACTN4, encoding $\alpha$-actinin- 4 , cause familial focal segmental glomerulosclerosis. Nat Genet 2000; 24: 251-56

[50] Winn MP, Daskalakis N, Spurney RE, et al. Unexpected role of TRPC6 channel in familial nephrotic syndrome: does it have clinical implications? J Am Soc Nephrol 2006; 17: 378-87.

[51] Bakris GL. Slowing nephropathy progression: Focus on proteinuria reduction. Clin J Am Soc Nephrol 2008; 3: S1-10.

(C) Chan and Roth; Licensee Bentham Open.

This is an open access article licensed under the terms of the Creative Commons Attribution Non-Commercial License (http://creativecommons.org/licenses/by$\mathrm{nc} / 3.0 /$ ) which permits unrestricted, non-commercial use, distribution and reproduction in any medium, provided the work is properly cited. 